PURD-TH-93-06

SISSA- $/ 93 / 49-\mathrm{A}$

$\mathrm{DFPD} / \mathrm{TH} / 93 / 27$

\title{
Baryogenesis, Domain Walls and the Role of Gravity
}

\author{
Henry Lew ${ }^{(a)}$ ] and Antonio Riotto ${ }^{(b)(c)}$ 民 \\ (a) Physics Department, Purdue University, West Lafayette, IN 47907-1396, U.S.A. \\ (b) International School for Advanced Studies, SISSA, via Beirut 2-4, I-34014 Trieste, Italy. \\ (c) Istituto Nazionale di Fisica Nucleare, Sezione di Padova, I-35100 Padua, Italy.
}

\begin{abstract}
It has been recently speculated that global symmetries are broken by gravity. We propose a scenario for the generation of the baryon asymmetry in the early Universe in which the domain walls predicted by theories with discrete symmetries become unstable due to these Planck scale effects. The relative motion of the decaying walls can provide a mechanism for the departure from thermal equilibrium necessary to have a nonvanishing baryon asymmetry. In particular, we implement this idea within the frameworks of the Left-Right and Quark-Lepton symmetric models.
\end{abstract}

\footnotetext{
${ }^{1}$ email: lew\%purdd.hepnet@LBL.Gov

${ }^{2}$ email: riotto@tsmi19.sissa.it
} 


\section{Introduction}

With the emergence of a gauge theory of the strong and electroweak interactions of particle physics and the standard hot big bang model of cosmology it became possible to attempt to answer the fundamental question of why there exists a matter-antimatter asymmetry in the Universe. Over the years there have been a number of scenarios proposed to describe the baryon asymmetry of the Universe (BAU) [1]. Recent attempts include baryogenesis at the electroweak scale using just the standard model (SM) of particle physics [2]. Though this scenario is attractive, it nevertheless requires the anomalous interactions to be out of equilibrium after the completion of the electroweak phase transition (EWPT), otherwise the BAU generated at the Fermi scale would be washed out by sphalerons [2]. This requirement translates into an upper bound on the mass of the scalar Higgs particle already excluded by the present LEP lower bound on the Higgs mass [3]. Moreover, for such a scenario to work, the EWPT must be of the first order and proceed via bubble nucleation, whereas recent analysis seem to indicate that it is very weakly first order or even second order [4].

In this paper we propose a new mechanism to generate the BAU in two particle physics models which predict new physics beyond the SM. The two models that we will consider are the Left-Right (LR) [5] and the QuarkLepton $(\mathrm{q}-\ell)$ [6] symmetric models. Both of these models will have interesting phenomenology which hopefully can be tested in upcoming experiments in the near future.

The basic requirements for the generation of the BAU [7] are: (1) baryon number violation, (2) $C$ and $C P$ violation and (3) a departure from thermal equilibrium. The key ingredients in our proposed mechanism which are 
needed to satisfy these basic requirements are given by (i) the lepton number $(L)$ violating interactions of the right-handed neutrino, $\nu_{R}$, (ii) domain walls and (iii) the intrinsic $C$ and $C P$ violations of the models under consideration. The right-handed neutrinos are responsible for establishing an initial lepton number asymmetry. The lepton number asymmetry is then converted to a baryon number $(B)$ asymmetry via the sphaleron at electroweak energies. Since the LR and $\mathrm{q}-\ell$ models have discrete symmetries which are spontaneously broken, the formation of domain walls will result [8]. Percolation theory seems to indicate that an "infinite" domain corresponding to each vacuum will form and therefore an "infinite" domain wall of complicated topology will appear [9].

Stable domain walls pose a problem in the standard big bang scenario unless the discrete symmetry breaking scale is very low [10. One way to resolve this problem is to break the discrete symmetry explicitly and thereby making the domain wall unstable. Recently there has been speculation that discrete symmetries which are not gauged are broken by gravity and that even very tiny effects of gravity may suffice to make the domain walls cosmologically harmless [1]. We propose a scenario in which the discrete symmetry is broken by gravity so that the domain walls become unstable and eventually disappear. This will provide a mechanism for the departure from thermal equilibrium. We wish to stress that in our scenario the EWPT is not required to take place via bubble nucleation but might be harmlessly of the second order.

We first describe the details our mechanism for baryogenesis in the LR symmetric model [12] and then in the $\mathrm{q}-\ell$ symmetric model. 


\section{LR symmetic model}

The LR symmetric model of weak interactions is based on the gauge group

$$
S U(2)_{L} \otimes S U(2)_{R} \otimes U(1)_{B-L}
$$

supplemented by a discrete symmetry between the left and right sectors of the model. The fermion content of the model is given by

$$
\begin{aligned}
& F_{L} \sim(2,1)(-1), \quad F_{R} \sim(1,2)(-1), \\
& Q_{L} \sim(2,1)(1 / 3), \quad Q_{R} \sim(1,2)(1 / 3),
\end{aligned}
$$

where $F$ and $Q$ denote the leptons and quarks respectively. The Higgs sector is given by

$$
\begin{gathered}
\Delta_{L} \sim(3,1)(2), \quad \Delta_{R} \sim(1,3)(2), \\
\Phi \sim(2,2)(0) .
\end{gathered}
$$

The symmetry breaking goes as follows when the Higgs fields acquire nonzero vacuum expectation values $(\mathrm{VEVs})$ :

$$
\begin{gathered}
S U(2)_{L} \otimes S U(2)_{R} \otimes U(1)_{B-L} \\
\left\langle\Delta_{L}\right\rangle=0 \downarrow\left\langle\Delta_{R}\right\rangle \neq 0 \\
S U(2)_{L} \otimes U(1)_{Y} \\
\downarrow\langle\Phi\rangle \neq 0 \\
U(1)_{Q}
\end{gathered}
$$

where $Y=2 I_{3 R}+(B-L)$ and $Q=I_{3 L}+Y / 2$.

As the temperature in the early Universe drops to about $T \sim\left\langle\Delta_{R}\right\rangle$ a domain wall forms due to the breaking of the left-right symmetry $\Delta_{R} \leftrightarrow \Delta_{L}$ 
[13]. To avoid the problems associated with stable domain walls we will assume that gravity explicitly breaks the discrete LR symmetry. This is not as ad hoc as it sounds since we know the gravitational interaction exists and presumably when a satisfactory quantum theory of gravity becomes available then the explicit breaking becomes calculable in principle.

The domain walls separate the two different domains which were formed independently after the cosmological phase transition when the discrete symmetry was spontaneously broken. Just after the formation of the wall, there exists massive left-handed neutrinos in one domain while in the other domain the massive neutrinos are right-handed. Assuming that gravity lifts the vacuum degeneracy of the two domains then the true vacuum will expand while the false one shrinks. If we take the true vacuum to be the domain with the massive right-handed neutrinos then an excess of massive right-handed neutrinos will result as those originally massless right-handed neutrinos move from the false vacuum to the true one. This is the qualitative picture of our out-of-equilibrium scenario. We now proceed to give some quantitative details.

Consider the two domains, denoted by $\mathrm{L}$ and $\mathrm{R}$, on either side of the domain wall. They are the local minima of the Higgs potential which have their degeneracy broken by the following terms induced by gravity

$$
\frac{K}{M_{p}^{2}}\left(\Delta_{L}^{\dagger} \Delta_{L}\right)^{3}+\frac{J}{M_{p}^{2}}\left(\Delta_{R}^{\dagger} \Delta_{R}\right)^{3}+\text { higher order terms, }
$$

where $K \neq J$ and $M_{p}$ is the Planck scale. These terms do not prevent the formation of the domain walls at $T \sim\left\langle\Delta_{R}\right\rangle$ since the energy difference between the two almost degenerate vacua is of the order of $\alpha\left\langle\Delta_{R}\right\rangle^{4}$, where $\alpha \sim\left\langle\Delta_{R}\right\rangle^{2} / M_{p}^{2}$, and $\alpha \ll 0.2 \beta, \beta$ indicating the generic coefficient of the quartic terms in the potential $V\left(\left\langle\Delta_{R}\right\rangle,\left\langle\Delta_{L}\right\rangle\right)$ [14]. 
As a result of the presence of the terms in Eq. (6) the wall moves and the region of false vacuum, call it L, shrinks. The energy released by the decay of the wall is

$$
\Delta \rho \sim|K-J| \frac{\left\langle\Delta_{R}\right\rangle^{6}}{M_{p}^{2}},
$$

where we will assume that $|K-J|$ is of order one. The domain wall decays in the time 15

$$
t_{W} \sim \frac{\eta}{\Delta \rho} \sim \frac{M_{p}^{2}}{\left\langle\Delta_{R}\right\rangle^{3}},
$$

where $\eta \sim\left\langle\Delta_{R}\right\rangle^{3}$ is the mass per surface area of the wall. By requiring that the wall decays before the EWPT so that the anomalous $B$-violating interactions are still active gives

$$
\left\langle\Delta_{R}\right\rangle>\left(M_{p} T_{E W}^{2}\right)^{\frac{1}{3}} \sim 10^{8} \mathrm{GeV},
$$

where $T_{E W}$ is the EWPT temperature. (We also need to check that the domain walls disappear before a time $t_{0}=M_{p}^{2} / \eta$ so that the energy density and the cosmic microwave background radiation of the observed Universe are not significantly affected [10]. By requiring that $t_{W} \lesssim t_{0}$ gives $\left\langle\Delta_{R}\right\rangle^{3} \gtrsim \eta$.)

During this time the right-handed neutrino gets a Majorana mass, $m_{\nu_{R}} \sim$ $\left\langle\Delta_{R}\right\rangle$, from the Lagrangian

$$
\mathcal{L}_{Y u k}=h\left[\overline{F_{L}}\left(F_{L}\right)^{c} \Delta_{L}+\overline{F_{R}}\left(F_{R}\right)^{c} \Delta_{R}\right]+\text { H.c.. }
$$

The number density of the out-of-equilibrium right-handed neutrinos which are produced and consequently decay is of the order of

$$
n_{\nu_{R}} \sim \frac{\Delta \rho}{m_{\nu_{R}}} \sim \frac{\left\langle\Delta_{R}\right\rangle^{5}}{M_{p}^{2}} .
$$


Note that the time by which the domain walls disappear (see Eq. (8)) corresponds to a temperature of

$$
T \simeq \sqrt{\frac{M_{p}}{t_{W}}}=\left\langle\Delta_{R}\right\rangle \sqrt{\frac{\left\langle\Delta_{R}\right\rangle}{M_{p}}} .
$$

For $\left\langle\Delta_{R}\right\rangle \sim 10^{8} \mathrm{GeV}$ and $M_{p} \sim 10^{19} \mathrm{GeV}$, this temperature is about $300 \mathrm{GeV}$. Also note that this temperature is less than the temperature of the righthanded neutrinos, $T_{\nu_{R}} \sim\left\langle\Delta_{R}\right\rangle$, and hence we expect them to be away from their equilibrium distributions. This means that the out-of-equilibrium conditions due to the moving wall persists until about $300 \mathrm{GeV}$. Below this temperature equilibrium is re-established and $L$ is converted to $B$ via sphaleron processes which conserves $(B-L)[2]$.

The BAU can now be calculated from the lepton number asymmetry due to the decays of the right-handed neutrino, i.e.

$$
\begin{aligned}
& \nu_{R_{i}} \rightarrow F_{L_{j}}+\bar{\Phi} \\
& \nu_{R_{i}} \rightarrow \bar{F}_{L_{j}}+\Phi
\end{aligned}
$$

By using Eq. (11) the lepton number density is given by

$$
n_{L_{i}}-n_{\bar{L}_{i}}=\frac{\left\langle\Delta_{R}\right\rangle^{5}}{M_{p}^{2}} \epsilon_{i}
$$

where $\epsilon_{i}$ is the difference between particle-antiparticle branching ratios [16]

$$
\epsilon_{i}=\frac{1}{2 \pi\left(\lambda \lambda^{\dagger}\right)_{i i}} \sum_{j}\left(\operatorname{Im}\left[\left(\lambda \lambda^{\dagger}\right)_{i j}\right]^{2}\right) f\left(m_{\nu_{R_{j}}}^{2} / m_{\nu_{R_{i}}}^{2}\right) \text {, }
$$

where

$$
f(x)=\sqrt{x}\left[1-(1+x) \ln \left(\frac{1+x}{x}\right)\right]
$$


and the $\lambda$ 's denote the Yukawa couplings between right-handed and lefthanded neutrinos through the Higgs bi-doublet $\Phi$ and are assumed to be complex to give a source of $\mathrm{CP}$ violation.

At $T \sim T_{E W}$ the lepton number produced by right-handed neutrino decays is

$$
\begin{aligned}
L & =\frac{n_{L}-n_{\bar{L}}}{s} \\
& \sim \frac{\left\langle\Delta_{R}\right\rangle^{5}}{10^{2} T_{E W}^{3} M_{p}^{2}} \epsilon,
\end{aligned}
$$

where the entropy density, $s$, is given by $s \sim g_{* s} T^{3} \sim 10^{2} T^{3}$. Since the domain walls decay just before dominating the energy density of the Universe, $L$ is not overdiluted by the energy released during the domain wall decay.

A nonzero baryon number is then induced via the $(B-L)$ conserving sphaleron processes given by

$$
\begin{aligned}
B & =\kappa L \\
& \sim \kappa \frac{\left\langle\Delta_{R}\right\rangle^{5}}{10^{2} T_{E W}^{3} M_{p}^{2}} \epsilon,
\end{aligned}
$$

where $\kappa$ is a numerical factor of $\mathcal{O}(1)$ and can be easily calculated from Ref. [17]. Note that at $T_{E W} \ll\left\langle\Delta_{R}\right\rangle, S U(2)_{R}$ sphalerons are no longer active and thus they do not affect Eq. (18)

For $\left\langle\Delta_{R}\right\rangle \sim 10^{8} \mathrm{GeV}, T_{E W} \sim 10^{2} \mathrm{GeV}$ and $M_{p} \sim 10^{19} \mathrm{GeV}$ gives a baryon asymmetry of about $B \sim 10^{-6} \epsilon$. Since the present baryon asymmetry lies in the range $(4-5.7) \times 10^{-11}$ [19], $\epsilon$ has to be as large as $10^{-5}$, which can be obtained for reasonable values of the $\lambda$ 's, e.g. $\lambda \sim \mathcal{O}\left(10^{-2}\right)$.

\footnotetext{
${ }^{3} S U(2)_{R}$ sphalerons are crucial for the mechanism described in Ref. 18 where righthanded neutrinos are suppose to scatter off first-order phase transition bubbles in the framework of LR models.
} 
We also need to check that the initial baryon asymmetry generated by the domain walls and the decaying right-handed neutrinos is not erased by a combination of other lepton violating interactions and sphaleron processes [20] at temperatures below that corresponding to the disappearance of the domain walls (see Eq. (12)). The lowest dimension $L$-violating operator in the effective low energy theory is given by

$$
\mathcal{L}_{\Delta L=2}=\frac{m_{\nu_{L}}}{v^{2}} F_{L} F_{L} \Phi \Phi+\text { H.c. }
$$

where $m_{\nu_{L}}$ is the mass of the left-handed neutrino and $v$ is the electroweak breaking scale. The interaction rate of these $\Delta L=2$ processes is $\Gamma_{\Delta L=2} \simeq$ $m_{\nu_{L}}^{2} T^{3} /\left(\pi^{3} v^{4}\right)$. For the survival of the pre-existing asymmetry we require this interaction rate to be less than the expansion rate of the Universe, i.e., $H \sim T^{2} / M_{p}$, where $T$ is given by Eq. (12). This results in a bound on the $S U(2)_{R}$ breaking scale

$$
\left\langle\Delta_{R}\right\rangle \gtrsim \frac{\lambda^{8}}{\pi^{6} h^{4}} M_{p}
$$

where $\lambda$ is the Yukawa coupling for the Dirac mass term and $h$ is the one for the Majorana mass. So for conservative values of the couplings, e.g. $\lambda \sim 10^{-2}$ and $h \sim 10^{-1}$, the bound on $\left\langle\Delta_{R}\right\rangle$ can easily be made lower than $10^{8} \mathrm{GeV}$, consistent with the bound from the lifetime of the domain wall.

\section{The $q-\ell$ model}

The analysis for generating the BAU in $\mathrm{q}-\ell$ model is essentially the same as that in LR model. In the following we outline how the mechanism is applied to the $\mathrm{q}-\ell$ model. 
Consider the q- $\ell$ model described by the gauge group

$$
S U(3)_{\ell} \otimes S U(3)_{q} \otimes S U(2)_{L} \otimes U(1)_{X} .
$$

$\mathrm{SU}(3)_{q}$ is the usual colour group and $\mathrm{SU}(3)_{\ell}$ is its leptonic partner. In addition there is a discrete $Z_{2}$ symmetry between the quarks and leptons. The fermion content of the model is given by

$$
\begin{aligned}
F_{L} & \sim(3,1,2)(-1 / 3), \quad E_{R} \sim(3,1,1)(-4 / 3), \quad N_{R} \sim(3,1,1)(2 / 3), \\
Q_{L} & \sim(1,3,2)(1 / 3), \quad u_{R} \sim(1,3,1)(4 / 3), \quad d_{R} \sim(1,3,1)(-2 / 3) .
\end{aligned}
$$

The Higgs sector is given byt

$$
\begin{gathered}
\chi_{1} \sim(\overline{3}, 1,1)(-2 / 3), \quad \chi_{2} \sim(1, \overline{3}, 1)(2 / 3), \\
\Delta_{1} \sim(6,1,1)(4 / 3), \quad \Delta_{2} \sim(1,6,1)(-4 / 3), \\
\phi \sim(1,1,2)(1) .
\end{gathered}
$$

The symmetry breaking pattern can be summarised as follows:

$$
\begin{gathered}
S U(3)_{\ell} \otimes S U(3)_{q} \otimes S U(2)_{L} \otimes U(1)_{X} \\
\left\langle\Delta_{1}\right\rangle \downarrow\left\langle\chi_{1}\right\rangle \\
S U(2)^{\prime} \otimes S U(3)_{q} \otimes S U(2)_{L} \otimes U(1)_{Y} \\
\downarrow\langle\phi\rangle \\
S U(2)^{\prime} \otimes S U(3)_{q} \otimes U(1)_{Q}
\end{gathered}
$$

The $\mathrm{SU}(2)^{\prime}$ is an unbroken gauge symmetry. This gauge force is expected to be asymptotically free. In analogy with QCD, we assume that it confines

\footnotetext{
${ }^{4}$ In the minimal $\mathrm{q}-\ell$ model the Yukawa Lagrangian yields the tree-level mass matrix relations, $M_{u}=M_{e}$ and $M_{d}=M_{\nu}^{\text {Dirac }}$, where $u$ refers to the charge $2 / 3$ quarks, $e$ refers to the charged leptons etc. The latter mass relation is broken by the Majorana mass terms for the neutrinos. The former relation can be evaded, for example, by introducing an additional Higgs doublet.
} 
all the exotic $\mathrm{SU}(2)^{\prime}$ coloured states, so that at large distances only colour singlet states exist in the spectrum.

The Higgs scalars, $\Delta_{1,2}$, play an analogous role to that of $\Delta_{L, R}$ in the LR model. The right-handed neutrinos gain Majorana masses through the term

$$
\mathcal{L}_{Y u k}=h\left[\overline{N_{R}}\left(N_{R}\right)^{c} \Delta_{1}+\overline{d_{R}}\left(d_{R}\right)^{c} \Delta_{2}\right]+\text { H.c. },
$$

when $\Delta_{1}$ develops a nonzero $\mathrm{VEV}$ while the $\mathrm{VEV}$ of $\Delta_{2}$ remains zero. At the same time the lepton number symmetry is broken so that an initial lepton number asymmetry can be generated. Without $\Delta_{1,2}$, there will exist a global lepton number symmetry, unbroken at tree-level, even though the q- $\ell$ symmetry will be broken by $\left\langle\chi_{1}\right\rangle$. Note that even if $\left\langle\Delta_{1}\right\rangle \sim 10^{8} \mathrm{GeV}$, new physics in the form of exotic "leptons" may still be observable in the $100 \mathrm{GeV}$ to $10 \mathrm{TeV}$ region provided $\left\langle\chi_{1}\right\rangle \ll\left\langle\Delta_{1}\right\rangle$.

The domain wall picture in the $\mathrm{q}-\ell$ model [21] is somewhat different from that of the LR model. Consider the situation for the $q-\ell$ model just after wall formation. The domains on each side of the wall are described by

$$
\begin{aligned}
& \text { region } 1:\left\langle\Delta_{1}\right\rangle \neq 0, \quad\left\langle\Delta_{2}\right\rangle=0 \\
& \text { region } 2:\left\langle\Delta_{1}\right\rangle=0, \quad\left\langle\Delta_{2}\right\rangle \neq 0 .
\end{aligned}
$$

The particles in region 1 will be the charged-conjugated versions of those in region 2. When the massive right-handed neutrino passes the wall from region 1 to region 2 it becomes a massless right-handed d-quark with hypercharge $Y=-2 / 3$ and vice versa. Similarly a massive right-handed neutrino passing the wall from region 2 to region 1 will become a massless righthanded (anti-)d-quark with $Y=2 / 3$. When the discrete symmetry is broken by gravitational effects, so that $\left\langle\Delta_{1}\right\rangle \neq\left\langle\Delta_{2}\right\rangle$, the wall will move since one domain will shrink at the expense of the other. There will be an excess of 
right-handed neutrinos created from the conversion of right-handed (massless) d-quarks as they move from the false vacuum to the true one. The remaining scenario for baryogenesis then proceeds like that in the LR model.

\section{Conclusions}

In the present paper we have shown that the role of gravity might be crucial not only for rendering topological defects like domain walls cosmologically harmless, but also for generating the baryon asymmetry in the early Universe. The point is that Planck scale effects could introduce new interaction terms which explicitly break the global discrete symmetries of the Lagrangian at tree level. If this is the case, then domain walls, which appear when discrete symmetries are spontaneously broken, can decay at a temperature below the typical scale of the theory, providing the out-of-equilibrium condition necessary to have a nonvanishing $B$. This mechanism can be successfully applied to the LR and $\mathrm{q}-\ell$ symmetric models if the $\mathrm{R}$ and the $\mathrm{q}-\ell$ breaking scales are of the order of $10^{8} \mathrm{GeV}$ respectively. Moreover, in our scenario, the EWPT need not be first order.

\section{Acknowledgements}

It is a pleasure to express our gratitude to P. Catelan, G. Dvali, S. Matarrese and G. Senjanović for stimulating and enlightening discussions. This work was supported in part by a grant from the DOE. 


\section{References}

[1] For a review of various baryogenesis scenarios see, for example, E. W. Kolb and M. S. Turner, Ann. Rev. Nucl. Part. Sci. 33, 645 (1983); A. D. Dolgov, Phys. Rep. 222, 309 (1992); A. G. Cohen, D. B. Kaplan and A. E. Nelson, UCSD-PTH-93-02 preprint (1993), to appear in the Ann. Rev. Nucl. Part. Sci. 43.

[2] V. A. Kuzmin, V. A. Rubakov and M. E. Shaposhnikov, Phys. Lett. B 155, 36 (1985); A. I. Bocharev and M. E. Shaposhnikov, Mod. Phys. Lett. A 2, 417 (1987); A. I. Bocharev, S. V. Kuzmin and M. E. Shaposhnikov, Phys. Lett. B 244, 275 (1990).

[3] Review of Particle Properties, Particle Data Group, Phys. Rev. D45, S1 (1992).

[4] J. R. Espinosa, M. Quirós and F. Zwirner, CERN-TH preprint 6577/92 (1992); W. Buchmüller, Z. Fodor, T. Helbig and D. Walliser, DESY preprint 93-21 (1993).

[5] J. C. Pati and A. Salam, Phys. Rev. D10, 275 (1974); R. N. Mohapatra and J. C. Pati, Phys. Rev. D11, 566, 2558 (1975); G. Senjanović and R. N. Mohapatra, Phys. Rev. D12, 1502 (1975).

[6] R. Foot and H. Lew, Phys. Rev. D41, 3502 (1990); Nuovo Cim. A 104, 167 (1991); R. Foot, H. Lew and R. R. Volkas, Phys. Rev. D44, 1531 (1991).

[7] A. D. Sakharov, Pis'ma Zh. Eksp. Teor. Fiz. 5, 32 (1967); JETP Lett. 5, 24 (1967). 
[8] For a review of topological defects in cosmology see A. Vilenkin, Phys. Rep. 121, 263 (1985).

[9] D. Stauffer, Phys. Rep. 54, 1 (1979).

[10] Ya. B. Zel'dovich, I. Yu. Kobzarev and L. B. Okun, Zh. Eksp. Teor. Fiz. 67, 3 (1974) [Sov. Phys. JETP 40, 1 (1975)].

[11] B. Rai and G. Senjanović, ICTP IC-92-414 (1992) preprint.

[12] Some of the basic ideas used in our mechanism for baryogenesis have been discussed by R. Holdom, Phys. Rev. D28, 1419 (1983).

[13] R. N. Mohapatra and G. Senjanović, Phys. Rev. Lett. 42, 1651 (1979); Phys. Rev. D20, 3390 (1979).

[14] G. B. Gelmini, M. Gleiser and E. W. Kolb, Phys. Rev. D39, 1558 (1989).

[15] P. Sikivie, Phys. Rev. Lett. 48, 1156 (1982); A. Vilenkin, Phys. Rev. D23, 852 (1981).

[16] M. Fukugita and T. Yanagida, Phys. Lett. B 174, 45 (1986); M. Luty, Phys. Rev. D45, 455 (1992).

[17] J. Harvey and M. S. Turner, Phys. Rev. D42, 3344 (1990).

[18] J.-M. Frère, L. Houart, J. M. Moreno, J. Orloff and M. Tytgat, CERNTH 6747/92 preprint (1992).

[19] T. P. Walker, G. Steigman, D. N. Schramm, K. A. Olive and H. S. Kang, Astrophys. J. 281, 51 (1991).

[20] M. Fukugita and T. Yanagida, Phys. Rev. D42, 1285 (1990). 
[21] For a discussion of domain walls in quark-lepton symmetric models see H. Lew and R. R. Volkas, Phys. Rev. D47, 1356 (1993). 